Draft VERSion OCtOBER 2, 2018

Preprint typeset using $\mathrm{LATE}_{\mathrm{E}} \mathrm{X}$ style emulateapj v. 05/12/14

\title{
DETECTION OF PERSISTENT GAMMA-RAY EMISSION TOWARD SS433/W50
}

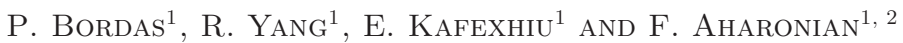 \\ ${ }^{1}$ Max-planck-Institut für Kernphysik, Saupfercheckweg 1, 69117 Heidelberg, Germany \\ 2 School of Cosmic Physics, Dublin Institute for Advanced Studies, 31 Fitzwilliam Place, Dublin 2, Ireland \\ Draft version October 2, 2018
}

\begin{abstract}
The microquasar SS433 features the most energetic jets known in our Galaxy. A large fraction of the jet kinetic power is delivered to the surrounding W50 nebula at the jet termination shock, from which high-energy emission and cosmic-ray production have been anticipated. Here we report on the detection of a persistent gamma-ray signal obtained with the Fermi Large Area Telescope from an unidentified source which we tentatively associate, given its $99.9 \%$ confidence level position accuracy and the lack of any other high-energy emitter counterpart in the studied region, with SS433. The obtained spectral energy distribution displays a distinct maximum at $\sim 250 \mathrm{MeV}$ and extends only up to $\sim 800 \mathrm{MeV}$. We discuss the possibility that the observed gamma-ray emission is produced through proton-proton collisions at the SS433/W50 interaction regions. If the same mechanism is operating in other baryon-loaded microquasar jets, their collective contribution could represent a significant fraction of the total galactic cosmic-ray flux at $\mathrm{GeV}$ energies.
\end{abstract}

ABSTRACT

Subject headings: Gamma rays: general — binaries: general - X-rays: binaries — stars: individual (SS433) — ISM: jets and outflows

\section{INTRODUCTION}

Evidences of significant gamma-ray emission from a few accreting X-ray binary systems (XRBs) have been collected in the recent years, owing to the improved capabilities of ground- and space-based gamma-ray instruments (see, e.g., Albert et al. 2007; Abdo et al. 2009; Ackermann et al. 2014). In all cases, such detections have been associated to transient phenomena, with gammaray emission thought to be produced in the interaction of energetic outflows with the surrounding matter and radiation fields. The kinetic energy carried by these outflows can increase significantly during such outbursts with respect to their quiescent state, with ejected kinetic luminosities reaching $L_{\text {kin }} \sim 10^{37-38} \mathrm{erg} \mathrm{s}^{-1}$, sustained for periods ranging from a $\sim$ few hours to some weeks (see, e.g., Mirabel \& Rodríguez 1994; Atoyan \& Aharonian 1999; Revnivtsev et al. 2002; Heinz \& Sunyaev 2002 and references therein).

SS433 is a well-known XRB system, and the first one in which highly energetic bipolar outflows (jets) were discovered (Abell \& Margon 1979; Fabian \& Rees 1979). Identified 30 years ago as a bright $\mathrm{H} \alpha$ line-emitting star close to the Galactic plane (Stephenson \& Sanduleak 1977) and as a non-thermal radio (Feldman et al. 1978) and X-ray source (Marshall et al. 1978), it has been since then object of intensive studies as an unusually bright and powerful microquasar (Mirabel \& Rodríguez 1999). The system is composed of a compact object, most probably a 10-20 $\mathrm{M}_{\odot}$ black hole (Gies et al. 2002), orbiting every 13.1 days a most-probable $\sim 11 \mathrm{M}_{\odot} \mathrm{A} 3-$ $7 \mathrm{I}$ star in a circular orbit with radius $\sim 56 \mathrm{R}_{\odot}$ (Hillwig et al. 2004), and is distinguished by a continuous (non-transient) supercritical accretion regime onto the black hole, with an accretion rate $\sim 10^{-4} \mathrm{M}_{\odot} \mathrm{yr}^{-1}(?)$, with $\mathrm{M}_{\odot}$ and $\mathrm{R}_{\odot}$ being the mass and radius of the sun,

pol.bordas@mpi-hd.mpg.de, ryang@mpi-hd.mpg.de respectively. A supercritical accretion disk is therefore formed (Shakura \& Sunyaev 1973), which feeds two subrelativistic jets carrying a kinetic energy power at a level of $L_{\text {kin }}^{\text {jet }} \sim 10^{39} \mathrm{erg} \mathrm{s}^{-1}$ (Dubner et al. 1998), ejected at a velocity $v_{\mathrm{j}}=0.26 \mathrm{c}$ (Margon \& Anderson 1989), where $c$ is the speed of light. Both the jets and the disk are observed to precess every 162.4 days in cones of half opening angle $\theta_{\text {prec }} \approx 21^{\circ}$ with respect to the normal to the orbital plane, which in turn subtends an angle $i \approx 78^{\circ}$ to our line of sight (Eikenberry et al. 2001). In addition to the extremely large jet power, SS433 represents also one of only two cases in which the presence of baryonic material in the jets has been established (the other source being 4U 1630-47, Díaz Trigo et al. 2013) through the detection of lines of highly ionized heavy elements (Marshall et al. 2002; Migliari et al. 2002). Clouds of plasma containing both electrons, ions and protons propagate along ballistic trajectories whilst radiating intensively in radio, optical and X-rays. Eventually, the jets get decelerated through their interaction with the ambient medium through the formation of a termination shock. Most of the jet kinetic power is delivered at the shock interface to the surrounding W50 nebula, a large $2^{\circ} \times 1^{\circ}$ size supernova remnant, SNR G39.7-2.0 (Green 2009), located at a distance of $\sim 5 \mathrm{kpc}$ (Fabrika 2004). Radio to X-ray emission originated in the jet/nebula interaction sites has been proposed (Safi-Harb \& Ögelman 1997; Dubner et al. 1998), whereas gamma-ray radiation from these regions has also been predicted (Heinz \& Sunyaev 2002), peaking at energies $\sim \Gamma_{\mathrm{j}} m_{\mathrm{p}} c^{2}$, where $\Gamma_{\mathrm{j}}=\left(1-v_{\mathrm{j}}^{2} / c^{2}\right)^{-1 / 2}$ is the bulk motion jet Lorentz factor. The detection of such a spectral feature would be a strong indication of cosmic-ray production in microquasar jets (Heinz \& Sunyaev 2002). Very-high-energy gamma-ray emission has also been proposed (Aharonian \& Atoyan 1998; Bosch-Ramon et al. 2005; Bordas et al. 2009) under the assumption that particle acceleration at 
the jet termination shock extends to TeV energies. No evidences of gamma-ray emission from SS433 has been reported so far (see, e.g., Aharonian et al. 2005; Hayashi et al. 2009; Saito et al. 2009).

\section{FERMI-LAT OBSERVATIONS AND ANALYSIS RESULTS}

We have analysed archival Fermi-LAT observations, spanning about five years of data-taking (from MJD 54,682.6 to MJD 56,719.4), corresponding to observations of a $20^{\circ} \times 20^{\circ}$ region centred on SS433 $\left(\mathrm{RA}=19^{\mathrm{h}} 11^{\mathrm{m}} 49^{\mathrm{s}} .57\right.$, Dec. $\left.=04^{\circ} 58^{\prime} 57^{\prime \prime} .9\right)$. This analysis has revealed the presence of a highly significant $(>7 \sigma)$ point-like source spatially coincident, within the $3 \sigma(99.9 \%)$ confidence level position accuracy of this newly discovered gamma-ray source, with the catalogued position of SS433. The data have been processed through the standard LAT analysis software together with the latest version of the instrument response functions as well as the Galactic and isotropic diffuse models provided by the Fermi-LAT Collaboration ${ }^{1}$. The sources present in the $3^{\text {rd }}$ Fermi-LAT catalogue were included in the analysis, while allowing for a variation of the point-source parameters in the likelihood fit for sources inside the studied region. A strong gamma-ray excess was apparent in the obtained residual maps. We use gtfindsrc to derive the best fit position of this excess. The best fit position is $\left[\mathrm{RA}=287.65^{\circ}\right.$, Dec $=4.72^{\circ}$, or about $0.41^{\circ}$ away from SS433's nominal position, with a $1 \sigma$ error circle of $0.15^{\circ}$. The residuals showed in addition the presence of another uncatalogued point-like source candidate in the FoV, labeled "ps2" hereafter, with best-fit position $\left[\mathrm{RA}=289.50^{\circ}, \mathrm{Dec}=6.09^{\circ}\right]$.

The inclusion of these two sources in the likelihood analysis, allowing again for a variation of their spectral parameters, provides a Test Significance value TS $=57.6$ above $200 \mathrm{MeV}$ (or about $7.3 \sigma$ ) for the source encompassing $\mathrm{SS} 433$, whereas a $\mathrm{TS}=52.0$ (or $\sim 6.9 \sigma$ ) is obtained for "ps2" in our analysis. A $5^{\circ} \times 5^{\circ}$ TS map of the region of interest (ROI) is shown in Fig. 1, together with the SS433 position and the $68 \%, 95 \%$ and $99.9 \%$ confidence contours on the position of the gamma-ray source enclosing it. Given the Point Spread Function (PSF) of the Fermi-LAT at energies $E_{\gamma} \sim 300 \mathrm{MeV}$, above $\sim 1.5^{\circ}$, the $0.41^{\circ}$ difference of the centroid of the gamma-ray signal with respect to SS433 coordinates, and the error circle radius of the source position, of $\sim 0.15^{\circ}$, the observed spacial coincidence prompts us for a tentative association of this new source with SS433.

A distinct feature of this detection is that the source shows a very unusual spectrum as compared to most of the Fermi-LAT catalog objects, with a spectral energy distribution (SED) displaying a maximum at $\sim 250 \mathrm{MeV}$, and extending only up to $\sim 800 \mathrm{MeV}$. The gamma-ray data is best fitted with a logparabola model, $N_{0}\left(E / E_{\mathrm{b}}\right)^{-\left(\alpha+\beta \log \left(E / E_{\mathrm{b}}\right)\right.}$, with $N_{0}=1.3 \pm 0.1 \times$ $10^{-7} \mathrm{~cm}^{-2} \mathrm{~s}^{-1} \mathrm{GeV}^{-1}, \alpha=0.010 \pm 0.004, \beta=1.9 \pm 0.1$, and $E_{\mathrm{b}}=0.182 \pm 0.005 \mathrm{GeV}$, yielding a Likelihood Test Ratio against a simple power-law of LTR $=-2 \mathrm{log}$ $\left(L_{\mathrm{PL}} / L_{\text {logpar }}\right)=13.2$. A cutoff power-law also fits well

\footnotetext{
${ }^{1}$ Software package v9r33p0, with the P7rep 15 version of postlaunch instrument response functions (IRFs). Fermi Science Support Center (http://fermi.gsfc.nasa.gov/ssc)
}

the data, with a value LTR $=8.3$ over a simple power-law fit.

The bolometric gamma-ray flux above $200 \mathrm{MeV}, \Phi_{\gamma}=$ $2.4 \pm 0.3 \times 10^{-8} \mathrm{ph} \mathrm{cm}^{-2} \mathrm{~s}^{-1}$, does not show any significant variability in the $\sim$ five-year data-set. We also searched for a possible periodic signature by folding the gamma-ray light-curve both on the orbital and precession periods of SS433. Due to the limited statistics, we divided all the available data in five equally spaced phasebins in both cases. The flux has been derived by applying a likelihood fit above $200 \mathrm{MeV}$ in each phase bin. The resulting light-curves, displayed in Fig. 2, are compatible with a constant flux. To constrain the possible contamination of the signal by the bright, nearby Fermi-LAT pulsar 3FGL J1907.9+0602, we used the Tempo2 pulsar timing package (Hobbs et al. 2006) and the latest ephemeris available for $\mathrm{J} 1907.9+0602^{2}$, and performed the same likelihood analysis but using a restricted dataset including only photons falling in the off-pulse emission period of J1907.9+0602. Using this $\sim 1 / 2$ exposure data-set, the source we associate to SS433 is detected at a TS value of 33 above $200 \mathrm{MeV}$. Both the total flux and the spectral properties are in complete agreement with respect to the analysis of the whole data-set, and we therefore make use of all available photons in the spectral analysis. The SED of the source associated to SS433 is shown in Fig. 3.

\section{DISCUSSION}

The location of the newly discovered gamma-ray source, the lack of any other high-energy counterpart in the studied region, and the extreme energetics and known non-thermal activity of SS433, argue for an association of the two sources.

Assuming a distance to SS433 of $d=5 d_{5} \mathrm{kpc}$, the derived flux translates to a gamma-ray luminosity $L_{\gamma} \sim$ $7 \times 10^{34} d_{5}^{2} \mathrm{erg} \mathrm{s}^{-1}$, which is much lower than the bolometric luminosity of the system, $L_{\mathrm{bol}} \sim 10^{40} \mathrm{erg} \mathrm{s}^{-1}$ (Fabrika 2004). The total power required to sustain it could be supplied close to the compact object by the supercritical accretion disk, or by the powerful jets both at the jet base or in the region of interaction with the W50 nebula. Moreover, several emission mechanisms could contribute significantly to the gamma-ray flux.

In a leptonic scenario, gamma-rays could be produced by Inverse Compton (IC) up-scattering off photons from the optical star and accretion disk or through relativistic Bremsstrahlung of electrons embedded in the cold proton/ion jet plasma or matter from the surrounding medium. We consider in the following a simple powerlaw distribution for relativistic electrons with an exponential cutoff, $N_{\mathrm{e}} \propto E_{\mathrm{e}}^{-\alpha_{\mathrm{e}}} \exp \left(-E_{\mathrm{e}} / E_{\mathrm{e}, \text { cut }}\right)$. More complex distributions for the emitting particles, e.g. a broken power-law, would require a proper knowledge of the acceleration conditions, possibly accounting for radiation or escape/adiabatic losses, which is beyond the scope of this study. Such a simple power-law distribution can fit the gamma-ray SED by assuming a hard electron spectrum in order to account for the low-energy data, at $E \lesssim 250 \mathrm{MeV}$. Taking $\alpha_{\mathrm{e}}=1.5$, the best fit is obtained for a cutoff energy $E_{\text {e,cut }} \approx 4.5$ and $1.0 \mathrm{GeV}$ in a IC and

${ }^{2}$ Fermi Pulsar Timing (http://www.slac.stanford.edu/ kerrm/fermi_pulsar_ti 
relativistic Bremsstrahlung scenario (see Fig. 3), providing a $\chi^{2} /$ d.o.f $=4.99 / 3$ and $2.37 / 3$, respectively. Given the smooth logparabola best-fit to the gamma-ray data, we also tried sub/super exponential cutoffs in the electron distribution, with $\exp \left(-E_{\mathrm{e}} / E_{\mathrm{e}, \text { cut }}\right)^{\beta}$ and $\beta \in[0,2]$, but no appreciable improvement in the fit is obtained.

If the emitter is located inside or close to the binarysystem, however, periodic absorption features are expected to affect the observed gamma-ray flux every precession cycle (see, e.g., Reynoso et al. 2008). For IC on the companion star photon field, an additional orbital modulation of the gamma-ray flux is expected given the high inclination of the system $\left(i \approx 78^{\circ}\right)$. No signature of such modulation is observed neither in the precession nor the orbital phase-folded light-curves (see Fig. 2), although the low statistics available prevent from a definitive conclusion.

If the emitter is instead located farther away along the jet, e.g. at the jet/medium interaction regions, IC on the cosmic microwave background would require a narrow electron distribution, approaching a delta function with $\gamma_{\mathrm{e}} \sim[3-5] \times 10^{5}$, to account for the observed gamma-ray flux. Relativistic Bremsstrahlung, on the other hand, could explain the observed emission by assuming target particle densities of $4 \times n_{\text {ion }} \approx 4 \mathrm{~cm}^{-3}$, with $n_{\text {ion }} \approx 1 \mathrm{~cm}^{-3}$ and a factor 4 from the RankineHugoniot jump conditions in strong shocks, and a relatively high efficiency, of about $\sim 30 \%$, in converting jetkinetic energy to non-thermal energy . Such estimate for the non-thermal efficiency is nonetheless strongly dependent on the total energetic budget of the emitter, which is in any case highly uncertain, and therefore unsuitable to extract more robust conclusions.

In a hadronic scenario, gamma-ray emission could be rendered through the decay of neutral pions produced in proton-proton collisions within the jet or at the jet termination site. The presence of a low-energy cutoff in the gamma-ray spectrum could be naturally explained in this scenario by the sharp decrease of the neutral pion production cross section close to the kinetic threshold, whereas the narrow gamma-ray peak could be easily described by adopting a proton distribution which does not extend significantly beyond a few GeVs. We have employed the latest parameterisation of the $p p \rightarrow \gamma$ cross sections given in Kafexhiu et al. (2014), which is valid for proton energies from kinematic threshold up to $\mathrm{PeV}$ energies. Assuming for simplicity a power-law distribution $N_{\mathrm{p}} \propto E_{\mathrm{p}}^{-\alpha_{\mathrm{p}}} \exp \left(-E_{\mathrm{p}} / E_{\mathrm{p}, \text { cut }}\right)$ for relativistic protons, the SED can be well fit with best parameters $\alpha_{\mathrm{p}} \approx 1.9$ and $E_{\mathrm{p}, \text { cut }} \approx 4.2 \mathrm{GeV}$. We note that a similarly peaked gamma-ray spectrum, extending only up to $\sim 1 \mathrm{GeV}$, has been previously reported from the observations of solar flares with the Fermi-LAT (Ackermann et al. 2014), for which an hadronic has been favoured.

The jets of SS433 could naturally provide such a proton distribution, with related gamma-ray emission having been anticipated as a result of the jets interaction with the surrounding medium (Heinz \& Sunyaev 2002). A total energy $L_{\text {kin }}^{\text {jet }} \times t_{\mathrm{SS} 433} \sim 6.3 \times 10^{50}$ ergs has been released by SS433 jets during the source life-time, $t_{\mathrm{SS} 433} \sim 2 \times$ $10^{4}$ yrs. A fraction of this energy, $W_{\mathrm{p}}$, is expected to be thermalized when the cold jet particles cross the strong shock formed at the jet/medium interface, or transferred to a shock-accelerated particle population. The collision of protons behind the termination shock against target nuclei from the shocked jet/medium shell could lead to gamma-ray emission through $\pi^{0}$ production and decay, with luminosities $L_{\gamma}^{\mathrm{pp}} \sim W_{\mathrm{p}} / t_{\mathrm{pp}} \mathrm{erg} \mathrm{s}^{-1}$. For $\sim 1 \mathrm{GeV}$ protons the $p p$ interaction time-scale is $t_{\mathrm{pp}} \approx 1.2 \times 10^{15} \mathrm{~s}$ for target particle densities of $4 \mathrm{~cm}^{-3}$. For the observed gamma-ray luminosity $L_{\gamma} \sim 7 \times 10^{34} \mathrm{erg} \mathrm{s}^{-1}$, setting $L_{\gamma}=L_{\gamma}^{\mathrm{pp}}$ implies that a moderate $\sim 10 \%$ fraction of the total accumulated energy should be contained in the injected protons and confined within the interaction regions. Assuming that the confinement time is defined by diffusion, with $D(E) \approx 3 \times 10^{27}\left(E_{\mathrm{p}} / 1 \mathrm{GeV}\right)^{0.5} \mathrm{~cm}^{2} \mathrm{~s}^{-1}$, and considering a length-scale for the interaction regions of $\Delta r \sim 10^{20} \mathrm{~cm}$, the diffusion time-scale is $t_{\mathrm{diff}} \sim$ $(\Delta r)^{2} / 2 D\left(E_{\mathrm{p}}\right) \approx 5 \times 10^{4} \mathrm{yr}$, which is larger than the source age. Note however that the estimations above assume that the source spectrum is primarily hadronic. An underlying contribution from Bremsstrahlung/IC to the observed flux cannot be ruled out, which would relax to some extent the energy requirements for the hadronic model.

The thermalization of the jet proton's kinetic energy at the termination shock provides a Maxwell-Boltzmann distribution peaking at $k_{\mathrm{B}} T \sim\left(\Gamma_{\mathrm{j}}-1\right) m_{\mathrm{p}} c^{2} \sim$ $35 \mathrm{MeV} /$ nucleon for the sub-relativistic jets in $\mathrm{SS} 433$ (with $\Gamma_{j}=1.036$ ). Even accounting for the interactions of protons in the high-energy tail of this thermal distribution, such a temperature would be insufficient to fit the $\geq 250 \mathrm{MeV}$ data-points of the gamma-ray spectrum. An additional population is therefore required, which could be supplied by accelerated protons injected along the jet or at the jet termination shock. The acceleration mechanism should be on the other hand rather inefficient, given that the proton spectrum should extend only up to a few GeVs. If acceleration takes place at the base of the jet, i.e., close to the compact object, these protons could emit gamma-rays through interactions with cold jet material or matter entrained from the disk wind. However, absorption of gamma-rays through $\gamma N$ interactions (Reynoso et al. 2008) should be apparent due to the periodic eclipses by the companion star and the precession of the accretion disk. Again, those absorption features are not observed in the phase-folded light-curves, within statistics (see Fig. 2). A steady flux is expected instead if gamma-rays are produced by protons accelerated at the interaction shock of the jets with the surrounding W50 nebula.

The tentative association of the steady gamma-ray flux reported here with the microquasar SS433 may have only been possible given the extreme kinetic power of its jets, $L_{\mathrm{kin}}^{\mathrm{SS} 433} \sim 10^{39} \mathrm{erg} \mathrm{s}^{-1}$. For the same efficiency and surrounding medium gas density, any steady gamma-ray emission and cosmic-ray production from other less powerful microquasars will scale down in flux by a factor $\sim\left(L_{\mathrm{kin}}^{\mathrm{SS} 433} / L_{\mathrm{kin}}^{\mu Q}\right) d_{5}^{-2} \sim 10^{2}-10^{3} d_{5}^{-2}$, being undetectable by current gamma-ray instruments. However, the cumulative contribution from the microquasar population in our Galaxy, possibly much larger than the $\sim 20$ systems identified so far (Fender 2006), could account for a measurable fraction of the galactic cosmic-ray flux at $\sim \mathrm{GeV}$ energies (Heinz \& Sunyaev 2002) if jets from other systems are also baryon-loaded (Díaz Trigo et al. 2013). 
Furthermore, the jets of established microquasars have Lorentz factors in the range $\Gamma_{\mathrm{j}} \sim 1-10$. Collective effects would make the combined gamma-ray peak to appear correspondingly broader and shifted-up to $\sim$ few GeVs. Interestingly, a pronounced spectral feature peaking at 1-4 GeV has been recently discovered in the inner regions of the Galaxy, extending for about 2-3 kpc from the Galactic Center (Hooper \& Slatyer 2013). Galactic jets could therefore be contributing to this $\mathrm{GeV}$ feature, which has been otherwise interpreted in a annihilating dark matter scenario (Hooper \& Goodenough 2011). Further studies on the spatial and spectral distribution of a yet unresolved gamma-ray emitting microquasar population are required to test this hypothesis.

Finally, we have considered so far gamma-rays being produced either at the jet base or in their interaction with the surrounding W50 nebula. The observed fluxes could be otherwise originated by the W50 remnant itself. The Fermi-LAT has indeed detected about 20 gamma-ray emitting SNRs (Fermi-LAT Collaboration 2015). Such emission has been claimed to have an hadronic origin in at least two systems, W44 and IC443 (Ackermann et al.
2013). Both sources are rather bright, with gamma-ray luminosities above $\sim 10^{-10} \mathrm{erg} \mathrm{cm}^{-2} \mathrm{~s}^{-1}$, and display spectral breaks at energies of $\sim$ few $\times 100 \mathrm{MeV}$, interpreted as the result of interactions with nearby molecular clouds. Despite their much broader spectra, extending well beyond the maximum $\sim 800 \mathrm{MeV}$ reported here $(\mathrm{IC}$ 443 , in particular, is detected at $\mathrm{TeV}$ energies, Albert et al. 2007), this could be explained by a more efficient proton acceleration occurring in these systems with respect to W50. The factor $\sim 10$ times higher gamma-ray luminosity displayed by W44 and IC 443 could be on the other hand accounted for given their relative closer location, at 2.9 and $1.5 \mathrm{kpc}$, respectively, compared to the $5 \mathrm{kpc}$ distance to W50.

Acknowledgments The authors are grateful to Stanislav Kelner, Anton Prosekin, Andrea Tramacere, Yasunobu Uchiyama and Andrew M. Taylor for fruitful discussions during this work. The authors are grateful as well to the anonymous referee, whose suggestions greatly helped to improve the manuscript. The authors acknowledge the Max-PlanckInstitut für Kernphysik financial support and excellent working conditions during this research.

\section{REFERENCES}

Abdo, A. A., Ackermann, M., Ajello, M., et al. 2009, Science, 326, 1512

Abell, G. O., \& Margon, B. 1979, Nature, 279, 701

Ackermann, M., Ajello, M., Allafort, A., et al. 2013, Science, 339, 807

Ackermann, M., Ajello, M., Albert, A., et al. 2014, ApJ, 787, 15

Aharonian, F., Akhperjanian, A., Beilicke, M., et al. 2005, A\&A, 439, 635

Aharonian, F. A., \& Atoyan, A. M. 1998, New AR, 42, 579

Albert, J., Aliu, E., Anderhub, H., et al. 2007, ApJL, 664, L87

Atoyan, A. M., \& Aharonian, F. A. 1999, MNRAS, 302, 253

Bordas, P., Bosch-Ramon, V., Paredes, J. M., \& Perucho, M. 2009, A\&A, 497, 325

Bosch-Ramon, V., Aharonian, F. A., \& Paredes, J. M. 2005, A\&A, 432, 609

Díaz Trigo, M., Miller-Jones, J. C. A., Migliari, S., Broderick, J. W., \& Tzioumis, T. 2013, Nature, 504, 260

Dubner, G. M., Holdaway, M., Goss, W. M., \& Mirabel, I. F. 1998, AJ, 116, 1842

Eikenberry, S. S., Cameron, P. B., Fierce, B. W., et al. 2001, ApJ, 561, 1027

Fabian, A. C., \& Rees, M. J. 1979, MNRAS, 187, 13P

Fabrika, S. 2004, Astrophysics and Space Physics Reviews, 12, 1

Feldman, P. A., Purton, C. R., Stiff, T., \& Kwok, S. 1978, IAU Circular, 3258, 1

Fender, R. 2006, Jets from X-ray binaries, ed. W. H. G. Lewin \& M. van der Klis, 381-419

Fermi-LAT Collaboration. 2015, ArXiv e-prints, arXiv:1501.02003

Gies, D. R., Huang, W., \& McSwain, M. V. 2002, ApJL, 578, L67

Green, D. A. 2009, Bulletin of the Astronomical Society of India, 37,45
Hayashi, S., Kajino, F., Naito, T., et al. 2009, Astroparticle Physics, 32, 112

Heinz, S., \& Sunyaev, R. 2002, A\&A, 390, 751

Hillwig, T. C., Gies, D. R., Huang, W., et al. 2004, ApJ, 615, 422

Hobbs, G. B., Edwards, R. T., \& Manchester, R. N. 2006,

MNRAS, 369, 655

Hooper, D., \& Goodenough, L. 2011, Physics Letters B, 697, 412

Hooper, D., \& Slatyer, T. R. 2013, Physics of the Dark Universe, 2,118

Kafexhiu, E., Aharonian, F., Taylor, A. M., \& Vila, G. S. 2014, ArXiv e-prints, arXiv:1406.7369

Margon, B., \& Anderson, S. F. 1989, ApJ, 347, 448

Marshall, F. E., Mushotzky, R. F., Boldt, E. A., Holt, S. S., \& Serlemitsos, P. J. 1978, IAU Circular, 3314, 2

Marshall, H. L., Canizares, C. R., \& Schulz, N. S. 2002, ApJ, 564, 941

Migliari, S., Fender, R., \& Méndez, M. 2002, Science, 297, 1673

Mirabel, I. F., \& Rodríguez, L. F. 1994, Nature, 371, 46

-. 1999, A\&A Rev., 37, 409

Revnivtsev, M., Gilfanov, M., Churazov, E., \& Sunyaev, R. 2002, A\&A, 391, 1013

Reynoso, M. M., Christiansen, H. R., \& Romero, G. E. 2008, Astroparticle Physics, 28, 565

Safi-Harb, S., \& Ögelman, H. 1997, ApJ, 483, 868

Saito, T. Y., Zanin, R., Bordas, P., et al. 2009, ArXiv e-prints, arXiv:0907.1017

Shakura, N. I., \& Sunyaev, R. A. 1973, A\&A, 24, 337

Stephenson, C. B., \& Sanduleak, N. 1977, ApJS, 33, 459 


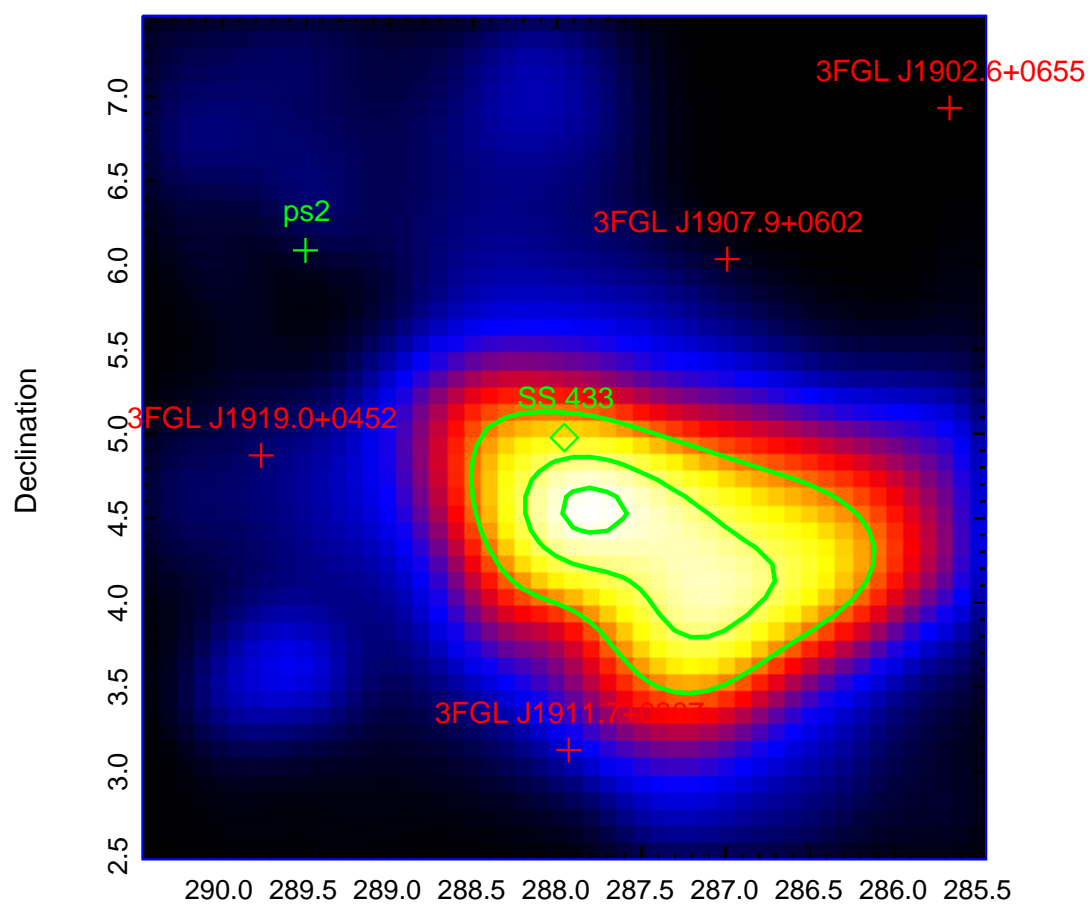

Right ascension

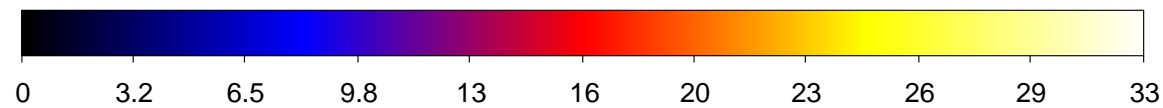

FIg. 1. - Test Significance (TS) map above $300 \mathrm{MeV}$ for the $5^{\circ} \times 5^{\circ}$ ROI around the nominal position of SS433, with pixel size corresponding to $0.1^{\circ} \times 0.1^{\circ}$. Sources included in the $3^{\text {nd }}$ Fermi-LAT catalog located within the ROI, in particular the bright gamma-ray pulsar 3FGL J1907+0602, as well as the candidate source "ps2" found in our analysis, have been excluded from the map for clarity. A green diamond indicates the position of SS433, whereas the green contours show the $68 \%, 95 \%$ and $99.9 \%$ positional accuracy of its proposed Fermi-LAT counterpart, with a TS value of 57.6.
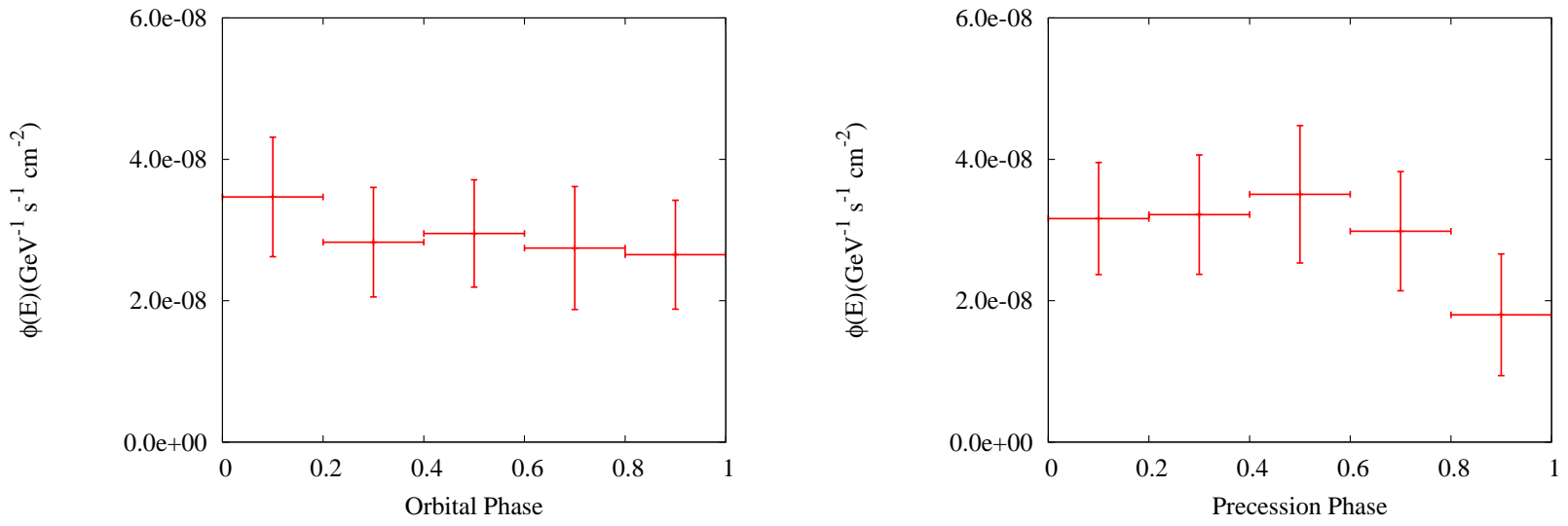

FiG. 2.- Phase-folded gamma-ray light-curve on the orbital and precession period of the system $(P=13.15$ and 162.4 days, respectively). Ephemeris by Gies et al. (2002) have been employed. No statistically significant signature of flux modulation is observed, with a constant fit to the data yielding $\chi^{2} /$ d.o.f of $0.60 / 4$ and $2.27 / 4$ for the orbital and precession phase-folded light-curves, respectively. 


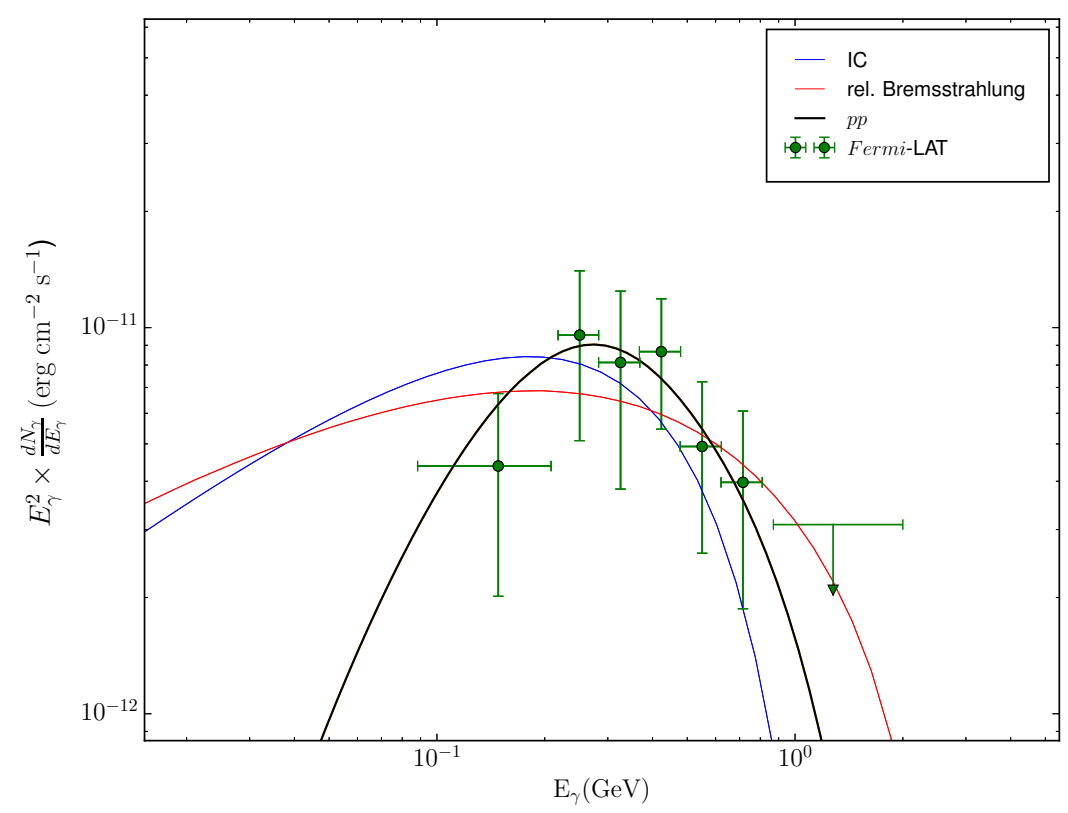

FIG. 3.- Spectral energy distribution of the Fermi-LAT flux together with fits to the gamma-ray emission considering emission produced through IC (blue), relativistic Bremsstrahlung (red) and proton-plasma interactions (black). The target photon field for IC is provided by the optical star and the accretion disk, $\left(T_{\star}=8500 \mathrm{~K}\right.$ and $T_{\text {disk }}=40.000 \mathrm{~K}$, respectively (Fabrika 2004)). Target material for relativistic Bremsstrahlung comes from cold jet particles or material entrained from the surrounding medium. An electron power-law distribution with $\alpha_{\mathrm{e}}=1.5$ and $E_{\text {cut }}=4.5(1.0) \mathrm{GeV}$ for IC (relativistic Bremsstrahlung) fits well the high-energy data points, but cannot reproduce the spectrum below $\sim 250 \mathrm{MeV}$. For $p p$ interactions, a thermal proton distribution with $k_{\mathrm{b}} T \sim 35 \mathrm{MeV}$ is insufficient to match the high-energy data-points. A proton power-law extending up to a few $\mathrm{GeVs}$ is required to fit the spectrum. 